\title{
Osteoclasts: the biological knife in sutural responses to mechanical stimulation
}

\author{
Mani Alikhani a,b,c, Sarah Alansarija,b, Mohammed M Al Jearah ${ }^{\mathrm{d}}$, Niraj Gadhavi ${ }^{\mathrm{a}}$, Mohammad A Hamidaddina, \\ Fadwa A Shembesh ${ }^{\mathrm{d}}$, Chinapa Sangsuwon ${ }^{\mathrm{a}, \mathrm{d}}$, Jeanne M Nervina ${ }^{\mathrm{a}, \mathrm{d}}$, Cristina C Teixeira ${ }^{\mathrm{a}, \mathrm{d}}$
}

\begin{abstract}
a Consortium for Translational Orthodontic Research, Hoboken, NJ

b Forsyth Institute, Cambridge, MA

c Department of Developmental Biology, Harvard School of Dental Medicine, Boston, MA

d Department of Orthodontics, New York University College of Dentistry, New York, NY
\end{abstract}

Corresponding Author: Cristina Teixeira, cristina.teixeira@nyu.edu

Citation: Alikhani M, Alansari S, Al Jearah MM, Gadhavi N, Hamidaddin MA, Shembesh FA, Sangsuwon C, Nervina JM, Teixeira CC. Innovation. April 2018. 1(4):e1. doi: $10.30771 / 2018.3$

Submitted April 8, 2018

Accepted April 9, 2018

Keywords: Expansion, Maxilla, Osteoclasts, Tensile stress, Sutures, Osteoblasts, Orthopedic Forces, Inflammatory Markers, Mechanical Stimulation, Bone Formation

\section{ABSTRACT}

Background: It is assumed that transverse force physically opens maxillary sutures and induces a tensile stress that directly stimulates bone formation. However, orthopedic/ orthodontic tensile stresses are static, which cannot directly stimulate bone formation. We hypothesize that the anabolic response to transverse force is indirect, resulting from inflammation-induced osteoclast activation followed by a transition into osteogenesis. The purpose of this study was to evaluate the tissue, cellular and molecular responses in the sutures during maxillary expansion.

Materials \& Methods: Adult Sprague-Dawley rats where divided into 4 groups: untreated Control (C), Sham (S), Experimental (Exp), and Experimental with nonsteroidal anti-inflammatory medication (Exp+NSAID). Maxillae were collected 0, 1, 3, 7, 14, and 28 days post-expansion for micro-computed tomography, fluorescence and light microscopy, gene and protein expression, and immunohistochemistry analysis.

Results: The Exp group showed early expression of inflammatory cytokines in the mid-palatal suture that was followed by osteoclast activation, bone resorption and a transient decrease in bone density. A significant widening of the suture occurred only after osteoclast activation and bone resorption. Bone formation was delayed, occurring after the initial catabolic phase. NSAIDs significantly decreased the magnitude of maxillary sutural widening and bone formation in response to transverse forces. During the transition from the catabolic to the anabolic response, expression of communicator molecules between osteoclasts and osteoblasts increased significantly.

Conclusion: We demonstrated that maxillary transverse force stimulated three distinct phases in the mid-palatal suture: 1) the Catabolic Phase starts with inflammatory markers and osteoclast recruitment and activity, 2) the Transition Phase, where osteoclasts activate osteoblast activity, 3) the Anabolic Phase, during which osteoblasts restore the integrity of the skeleton.

INNOVATION: Our findings are novel and support a new theory for sutural response to orthopedic forces that emphasizes the importance of osteoclasts in the process and makes these cells the possible target of orthopedic treatment: 1) as the "biological knife" at the sutures that allows the separation of maxillae for orthopedic movement, and 2) as the trigger for osteoblasts activation and bone regeneration, anytime we need to stimulate bone formation especially at the surface of the cortical bone. 


\section{Background}

Physiological mechanical loads are osteogenic for long bones and the craniofacial skeleton (1-3). Mechanical loading directly targets the mechanoreceptors in bone (osteocytes), which then stimulate the bone remodeling machinery (osteoclasts and osteoblasts) to adapt to the new load demand $(4,5)$. However, orthopedic forces on craniofacial bones (especially the maxilla) or orthodontic forces on teeth, are used clinically with different expectations. It is believed that application of compressive orthodontic or orthopedic forces stimulate bone resorption, while application of tensile forces stimulates bone formation. This is even more confusing since the nature of these orthopedic and orthodontic forces is static, meaning they are activated and allowed to dissipate until the next activation. Previous studies have demonstrated that direct application of static forces on long bones or alveolar bone does not have any significant effect on the skeleton $(3,6)$. How, then, can the model of bone resorption and formation due to static orthopedic and orthodontic forces be explained?

Comparing clinical examples of mechanical loading of weight bearing bones and the craniofacial skeleton shows that weight bearing bones load directly while loading craniofacial bones is mostly indirect, with an intermediary connective tissue between the point of force application and the bone. The connective tissue for the upper jaw (maxilla) is sutures that connect the maxilla to the rest of the skull. For the teeth, the connective tissue is the periodontal ligament (PDL) that separates the teeth from alveolar bone.

Recently, we and others (7-10) have shown that applying static force to the teeth via orthodontic forces stimulates an inflammatory reaction in the PDL that is critical for tooth movement. Specifically, the inflammatory response in the PDL activates osteoclasts in the PDL and adjacent alveolar bone to resorb alveolar bone, thereby allowing the teeth to move. Importantly, this biological response to static orthodontic forces is indirect, requiring the presence of the PDL. Once the orthodontic forces dissipate, bone resorption ceases and bone formation begins to maintain the integrity of alveolar bone.

While the static compression-induced inflammatory reaction observed in the PDL explains alveolar bone resorption during orthodontic treatment, the reasons for bone formation reported in the area of tensile force in the PDL or sutures are not clear. The widely-held explanation is that tensile force directly stimulates bone formation. But, the facts are that 1) tensile force that are applied during craniofacial orthopedic and orthodontic treatment are mostly static, 2) application of static tensile force in long bones does not cause bone formation (11), and 3) both tension and compression forces are osteogenic under conditions of high force frequency and acceleration (dynamic load) $(6,12$, 13) . These conflicting data led us to question the accuracy of the widely-held belief that there is a direct connection between tensile force and osteogenesis.

Since both compression and tension stresses that result from static orthodontic forces can traumatize the PDL (14), it is possible that bone formation observed in response to tensile orthopedic forces is a delayed reaction and not a direct effect of these forces on the bone. Therefore, one would expect that in response to tensile stresses, similar to compressive stresses, the osteoclasts would appear in the area of trauma, and since osteoclasts play a role in activating osteoblasts (15), it is possible that activating osteoclasts is a prerequisite for osteoblast activity observed in the area.

To test our hypothesis, we systematically examined the effect of maxillary expansion force on the mid-palatal suture in rats. Specifically, we examined the skeletal, cellular and molecular changes that occurred during the first 28 days of expansion treatment.

\section{Materials and Methods}

\section{Animals and Study Design}

Growing Sprague-Dawley male rats ( $\mathrm{n}=95$, average weight $60 \mathrm{~g}, 21$ days old) were treated according to the protocol approved by New York University Institutional Animal Care and Use Committee. Rats at this stage are in a growth phase and their first and second maxillary molars are fully erupted. All animals were housed in polycarbonate cages in a 12-h light/ dark environment at a constant temperature of $23^{\circ} \mathrm{C}$ and fed a standard pellet diet (expanded pellets; Stepfield, Witham, Essex, UK) with tap water ad libitum.

Animals were randomly divided into four groups: Control (C), Sham (S), Experimental (Exp), and Experimental with nonsteroidal anti-inflammatory drug (Exp + NSAID). The Control group animals did not receive any appliance or drug treatment. The Experimental group received a calibrated custom-designed expansion spring that delivered transverse force $(100 \mathrm{cN})$ to the first and second molars (Fig. 1). This force was selected based on previous studies that demonstrate that $100 \mathrm{cN}$ maximally induces cellular activity in maxillary sutures (16). Considering that the rats would have a normal vertical chewing force (averaging 54-76 N (17)), this force is not considered excessive. The appliance was connected to the first molars with ligature wires and fixed with composite. The Sham group
A

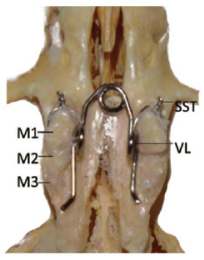

B

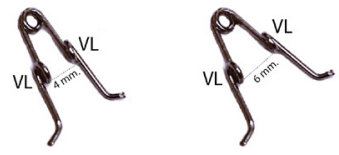

Figure 1: Calibrated springs used to produce transverse forces in the rat maxilla. Springs were fabricated from 0.016" stainless steel wires (3M Unitek, Monrovia, CA, USA), with two vertical loops used to tie the spring to the maxillary first molars using 0.008 " stainless steel ligature ties and composite. (A) Photograph of springs installed in the rat maxilla. (B) The springs were calibrated using a digital force gage to produce $100 \mathrm{cN}$ force when expanded from $4 \mathrm{~mm}$ (left) to $6 \mathrm{~mm}$ (right) $(V L=$ vertical loop, $S S T=$ stainless steel tie, $M 1=$ first molar, $M 2$ = second molar, M3= third molar). 
received a similar spring that was not activated and did not produce any force. The Experimental + NSAID group received a similar active spring as the Experimental group and a daily dose of a Diclofinac $(5 \mathrm{mg} / \mathrm{kg})$ administered intramuscularly (IM) with changing injection sites to prevent additional discomfort to the animals. Animals were weighed daily to accurately calculate the dose of the medication for each animal.

Bone labeling was performed by means of an intraperitoneal injection of calcein $(15 \mathrm{mg} / \mathrm{kg})$ on Day 0 , xylenol orange $(25 \mathrm{mg} /$ $\mathrm{kg})$ on Day 12 and demoxicycline $(25 \mathrm{mg} / \mathrm{kg})$ on Day 26 . The health status and body weight of the rats were evaluated daily, and no significant differences were observed among groups. Animals were euthanized by CO2 narcosis on Days $0,1,3$, 7,14 , and 28. Samples were collected for micro-CT analysis $(\mu \mathrm{CT})$, histology and immunohistochemistry ( 5 animals per time point, per group; total of 25), fluorescence microscopy (5 animals per group scarified on Day 28; total of 20), mRNA (5 animals per time point, per group; total of 25) and protein analysis (5 animals per time point, per group; total of 25).

The sample size was calculated based on the results of our previous animal studies (22). Assuming an estimated 50\% difference in the expression of inflammatory markers in the PDL, Type I error was set at 5\% and the power of the statistical test was set at $90 \%$ (power $=0.9, \alpha=0.1$ ). Based on these values, a sample size of 4 animals per group was calculated. We decided to increase the sample size to 5 to account for attrition.

\section{Transverse force application}

On Day 0, animals from the Sham, Expansion and Expansion + NSAIDs groups were anesthetized with an intraperitoneal injection of Ketamine and Xylazine $(0.09 \mathrm{~mL} / 100 \mathrm{~g})$. Anesthesia was verified by lack of response to toe-pinch. All groups were fitted with custom designed expansion springs. Springs were fabricated from 0.016 " stainless steel wires (3M Unitek, Monrovia, CA, USA), with two vertical loops used to tie the spring to the first maxillary molars using a 0.008 " stainless steel ligature ties. Springs were secured by placing a thin layer of composite on the interproximal surfaces of the first molars while avoiding the occlusal surface of the teeth (Figs. 1A and 1B). The springs were calibrated to produce $100 \mathrm{cN}$ force $(50 \mathrm{cN}$ on each side) using a digital force gage. Application of tensile forces was carried out for 0-28 Days as noted above. Animals and the integrity of the springs were monitored daily under inhalation anesthesia (isoflurane-nitrous oxide). If springs were dislodged they were reinstalled at this time.

\section{Specimen preparation}

$\mu \mathrm{CT}$, histology and immunohistochemistry analyses. The whole skull was dissected and fixed for 72 hours with $4 \%$ (w/v) paraformaldehyde in $0.1 \mathrm{M}$ phosphate buffer, $\mathrm{pH} 7.4$ followed by storage in $70 \%$ ethanol. $\mu \mathrm{CT}$ analysis was then performed, after which all samples were demineralized in $14 \%$ ethylenediaminetetraacetic acid (EDTA) for 3-4 weeks at $4{ }^{\circ} \mathrm{C}$ and dehydrated in ethanol gradients and xylene prior to embedding in paraffin.

Fluorescence microscopy. After fixation in $100 \%$ methanol for three days at $4{ }^{\circ} \mathrm{C}$, samples were placed in acetone for two days at room temperature. Specimens were then dehydrated in alcohol, cleared in xylene, and embedded in methyl methacrylate according to the method of Erben (18).

\section{Micro-CT Analysis}

Before decalcification, the whole maxilla was scanned by micro-computed tomography $(\mu \mathrm{CT})$ on a Skyscan 1172 system (Bruker microCT, Kontich, Belgium). The specimens were scanned in $70 \% \mathrm{ETOH}$ at $13.55 \mu \mathrm{m}$ voxel size, $100 \mathrm{KV}$, 0.300 o rotation step $(192.30$ o angular range) and a $1910 \mathrm{~ms}$ exposure. Results were analyzed utilizing NRecon software (1.6.9.16 version, Bruker micro-CT, Kontich, Belgium) for 3D reconstruction and viewing of images. Superimpositions were performed using Amira 6.0.0 software (FEI Houston Inc., Hillsboro, OR).

Three measurements were obtained from the $\mu \mathrm{CT}$ images at the level of the mid-coronal plane of the maxillary first molar (Fig. 2): 1) width of the mid-palatal suture (distance between the borders of the suture), 2) width of palate (distance between the palatal walls at the level of intersection between palate and alveolar), and 3) interdental width (distance between the height of contour of the first molars).

The bone volume change in the palate was measured to investigate the effects of transverse force on the mid-palatal suture and adjacent bone. The region of interest (ROI) for bone volume measurements in the palate included the mid-palatal suture extending from the first molar to the area between first and second molars, from the palatal concavity to the floor of the nasal cavity, and $0.5 \mathrm{~mm}$ laterally on each side of the suture midline $(2.5 \mathrm{~mm} \times 1 \mathrm{~mm} \times 0.5 \mathrm{~mm})$.

The second ROI was the inter-radicular bone of the first molar. This area was limited by a horizontal plane (parallel to the occlusal plane) passing through the alveolar crest, and a horizontal plane passing through the apex of the mesio-buccal root of the first molar. Anteriorly, the ROI was limited by the mesio-buccal root, posteriorly by the disto-buccal and disto-palatal roots, laterally by the inter-buccal and mesio-palatal roots.

Two examiners completed all $\mu \mathrm{CT}$ quantifications. Both intra-observer and inter-observer errors were evaluated. Intraobserver error was evaluated by individual investigators who measured $5 \mu \mathrm{CT}$ images twice at least 2 weeks apart. Interobserver error was evaluated using the same set of $5 \mu \mathrm{CT}$ images measured by a second investigator. The Dahlberg (19) formula was applied to estimate the random errors and the paired t-test was applied to identify systematic errors according to Houston (20). Random error for intra-observer evaluation was $0.015 \mathrm{~mm}$ and $0.017 \mathrm{~mm}$ for the inter-observer evaluation, and not statistically significant. Systematic errors were also small and not statistically significant ( $\mathrm{p}=0.87$ for intra-observer and $\mathrm{p}=0.84$ for inter-observer). 


\section{Histology and Immunohistochemistry}

Fixed demineralized maxillae were embedded in paraffin, and cut into $5 \mu \mathrm{m}$ occlusal sections, using a Leica Biosystems RM2265 Fully Automated Rotary Microtome (Leica Biosystems Inc., IL, USA). Some sections were stained with Hematoxylin and Eosin $(\mathrm{H} \& \mathrm{E})$ to view the general cellular and tissue structure. Consecutive sections were stained with TRAP (tartrate resistant acid phosphatase) to identify osteoclasts. Osteoclasts were defined as TRAP-positive multinucleated bright red cells in the mid-palatal suture or adjacent bone. Sections were scanned on a Scan Scope GL series optical microscope (Aperio, Bristol, UK) at 20X magnification. Osteoclasts were counted in five consecutive sections, in the area from mesial of the first molar to mesial of the second molar, including $1 \mathrm{~mm}$ of surrounding bone, and values were averaged for each rat. Data were expressed as the mean number of TRAP-positive cells per $1 \mathrm{~mm} 2$ area of suture and adjacent alveolar bone. Two examiners completed all histological quantifications.

\section{Fluorescence Microscopy}

Samples embedded in methyl methacrylate were trimmed and oriented on the Leica 2265 microtome (Leica Biosystems Inc., IL, USA), polished using gradients of polishing paper until smooth, loaded on to a plastic slide, cut to $100 \mu \mathrm{m}$ thickness, and polished down to $30 \mu \mathrm{m}$. Slides were then viewed by fluorescence microscopy and photographed (Nikon Microscopy, NIS-Elements software, Tokyo, Japan).

\section{RT-PCR Analysis}

For gene expression studies, 5 animals from each group were euthanized by CO2 narcosis at Days $0,1,3,7,14$, and 28. The palatal bone extending $0.5 \mathrm{~mm}$ from each side of the mid-palatal suture was dissected and frozen in liquid nitrogen. The alveolar bone surrounding the molars was also dissected and frozen for PDL studies. Total mRNA was isolated using TRIZOL reagent (Life Technologies, New York, NY, USA), and mRNA clean-up was performed with an RNeasy Mini Kit (Qiagen Sciences, Valencia, CA, USA) as described previously (21). All equipment and tools were cleaned with RNaseZap (Sigma, St. Louis, MO, USA). Twenty-nine inflammatory cytokines and cytokine receptor genes, osteoclast markers, osteoblast markers, and bone simulating factor, were analyzed with primers specific for rat genes, with a QuantiTect SYBR Green RT-PCR kit (both Qiagen, Valencia, CA, USA) on a DNA Engine Optican 2 System (MJ Research, Waltham, MA, USA). Each mRNA specimen was tested 3 times. Relative levels of mRNA were calculated and normalized to the level of GAPDH and acidic ribosomal protein mRNA.

\section{Protein Analysis}

Protein levels of inflammatory markers were measured by enzyme-linked immunosorbent assay (ELISA). Five palatal bones extending $0.5 \mathrm{~mm}$ from each side of the mid-palatal suture were dissected, frozen in liquid nitrogen and pulverized.
The alveolar bone surrounding the molars was also dissected and treated the same way for PDL studies as described previously (Teixeira et al., 2010). Lysates were prepared and total protein quantitated using a BCA protein assay kit (Pierce, Rockford, IL). Concentration of interleukin (IL)-1 $\beta$ (Thermo, Rockford, IL), tumor necrosis factor alpha (TNF- $\alpha$ ) (Thermo, Rockford, IL), CCL5 (Abnova, Walnut, CA), CCL2 (Abcam, Cambridge, MA), and RANKL (MyBioSource, San Diego, CA) were determined by ELISA. Data were analyzed in comparison to standard curves specific to each inflammatory marker.

\section{Statistical Analysis}

After confirming normal distribution of samples by the Shapiro-Wilk test, group comparisons were assessed by analysis of variance (ANOVA). Pairwise multiple comparison analysis was performed with Tukey's post-hoc test. In some experiments a paired t-test was used to compare the two groups. Two-tailed p-values were calculated; $\mathrm{p}<0.05$ was set as the level of statistical significance.

\section{Results}

\section{Increase in mid-palatal and circum-maxillary suture widths in response to transverse forces}

Applying transverse forces to the maxilla through a tooth-anchored expander is routinely used in Orthodontics. To understand the morphological changes observed in the mid-palatal and circum-maxillary sutures in response to transverse forces, we measured the width of the sutures, the palate and the dental width in three-dimensional images obtained by $\mu \mathrm{CT}$ (Fig. 2 A). One would expect that if the effect of transverse forces is purely physical, there should be a linear relationship between the magnitude of suture widening and the increase in palatal and dental widening. We found a significant increase in the mid-palatal suture width at Days 3, 7, 14 and 28 in the Experimental group compared to the Sham group $(\mathrm{p}<0.05)$ (Figs. 2B and 2C). After Day 14, the mid-palatal suture demonstrated a tendency to return to its original width, as shown by a significant decrease in width on Day 28.

Surprisingly, the dental and palatal widths in the Experimental group were only significantly increased on Day $14(0.89 \mathrm{~mm}$ and $0.54 \mathrm{~mm}$ increase, respectively) and Day 28 $(1.26 \mathrm{~mm}$ and $0.92 \mathrm{~mm}$, respectively) $(\mathrm{p}<0.05)$ compared to the Sham group. At both time points, the amount of dental change was higher than the skeletal change $(\mathrm{p}<0.05)$ (Fig. 2B). While the skeletal and dental changes were minimum at Days 1, 3 and $7(p>0.05)$, the increase in width of the suture at Days 3 and 7 was statistically significant, which demonstrates that sutural opening occurs ahead of skeletal and dental changes and they do not follow a linear relationship.

Applying transverse forces on the maxillary teeth not only increased the width of the mid-palatal suture but simultaneously affected the surrounding sutures, specifically the zygomaticotemporal, zygomaticomaxillary, frontomaxillary 
A
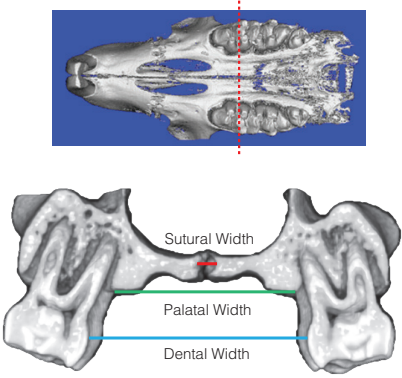

B

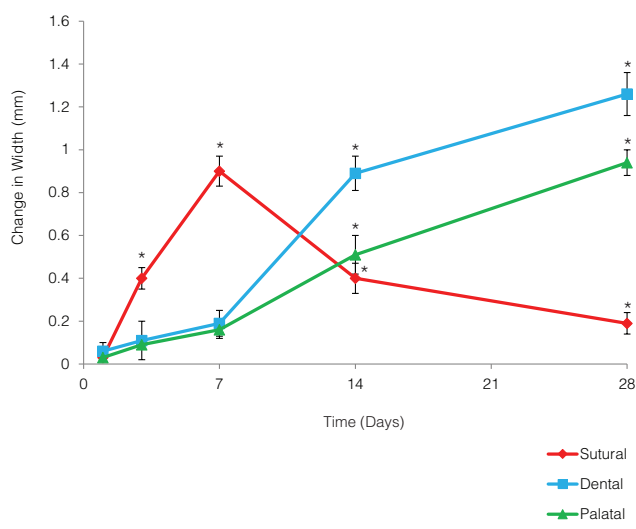

C

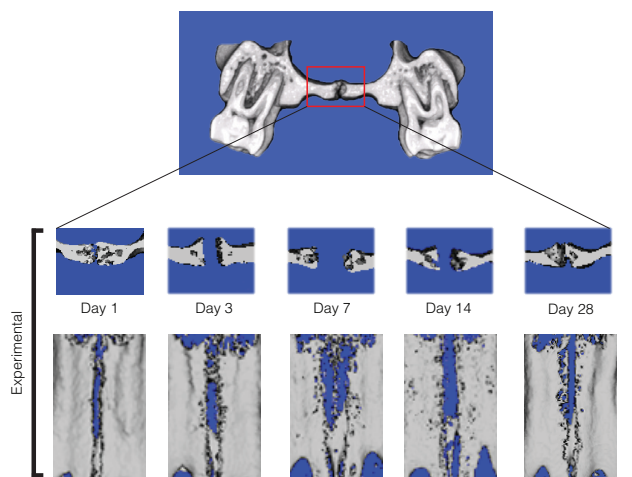

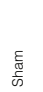

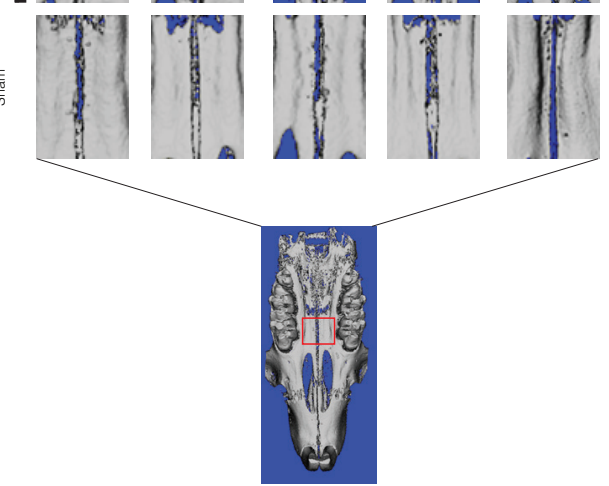

Figure 2: Changes in sutural, palatal and dental widths over time. (A) Palatal width, dental width and mid-palatal suture widths were measured using $\mu C T$ 3D reconstructed images and sections at the level of the mid-coronal plane of the maxillary first molar. Red line shows the width of the mid-palatal suture (distance between the borders of the suture), Green line shows the width of palate (distance between the palatal walls at the level of intersection between the palate and alveolar walls), and Blue line shows the dental width (distance between height of contour of first molars). (B) Sutural, palatal and dental widths were measured over time in the Experimental and Sham maxillae. Data expressed as the mean $\pm S D$ of distances in $\mathrm{mm}$. Each number represents the average of 5 samples. $\left({ }^{*}\right.$ Experimental width significantly different from Sham, $p<0.05)$. (C) Coronal (through the mid-coronal sections of the maxillary first molars) and occlusal (from oral cavity) $\mu C T$ images of the mid-palatal suture opening at different time points.

and transverse sutures (Figs. 3A and 3B). Similar to the changes observed in the mid-palatal suture, the width of these sutures in the Experimental animals on Days 3, 7, and 14 showed a statistically significant increase, with a peak increase at Day 7 ( $<<0.05)$ (Fig. 3C). Interestingly, the final increase in palatal width $(0.92 \mathrm{~mm})$ correlated closer with the maximum width of the zygomaticomaxillary ( 0.52 on each side) and zygomaticotemporal sutures $(0.51 \mathrm{~mm}$ on each side) than with the width of the transverse $(1.4 \mathrm{~mm})$, frontomaxillary $(1.1 \mathrm{~mm})$ or mid-palatal $(1.2 \mathrm{~mm})$ sutures.

\section{Cellular changes in the mid-palatal suture: increases in osteoclast number and activity following expansion}

To correlate the morphological changes we observed in the mid-palatal suture following expansion with cellular changes, we investigated the presence of osteoclasts by TRAP staining. Compared to the Sham group, the Experimental group developed a noticeable change in the suture's cellular organization on Days 7 and 14, which correlated with the greatest sutural widening observed in Figs. 2B and 2C. We observed an increase in the number of TRAP-positive osteoclasts, especially at Days
3, 7 and 14, in the mid-palatal suture and adjacent bone (Fig. $4 \mathrm{~A})$. For quantitative analysis, osteoclasts were counted in 5 consecutive sections in the area including the mid-palatal suture and surrounding bone. The number of osteoclasts in the Experimental group was significantly higher on Days 3,7 and 14 compared to the Sham group ( $\mathrm{p}<0.05$ ) (Fig. 4B).

To further evaluate the activity of osteoclasts in the mid-palatal suture, expression of the osteoclast markers RANK, OPG (osteoprotegerin), RANKL, and CtsK (Cathepsin K) was evaluated at different time points (Fig. 4C). RANK expression was significantly higher on Days 3, 7 and 14 (2.6-, 3.4- and 2.8-fold, respectively) $(p<0.05)$, but no differences were observed on Days 1 and $28(\mathrm{p}>0.05)$. OPG expression was significantly higher on Days 3, 7, and 14 (2.4-, 3.7- and 4.5-fold, respectively) in the Experimental group compared to the Sham group. No difference in OPG expression was observed on Days 1 and 28 $(\mathrm{p}<0.05)$. RANKL expression was significantly higher $(\mathrm{p}<0.05)$ on Days 1, 3, 7, and 14 (2.6-, 4.8-, 5.4-, 3.9-fold, respectively) in the Experimental group compared to the Sham group. CtsK expression at Days 1, 3, 7, and 14 (2.3-, 3.4-, 6.2-, and 5.1-fold, respectively) increased significantly $(\mathrm{p}<0.05)$ in the mid-palatal 
A

Zygomaticomaxillary Suture

Frontomaxillary Suture

Fronto-Maxillary Suture
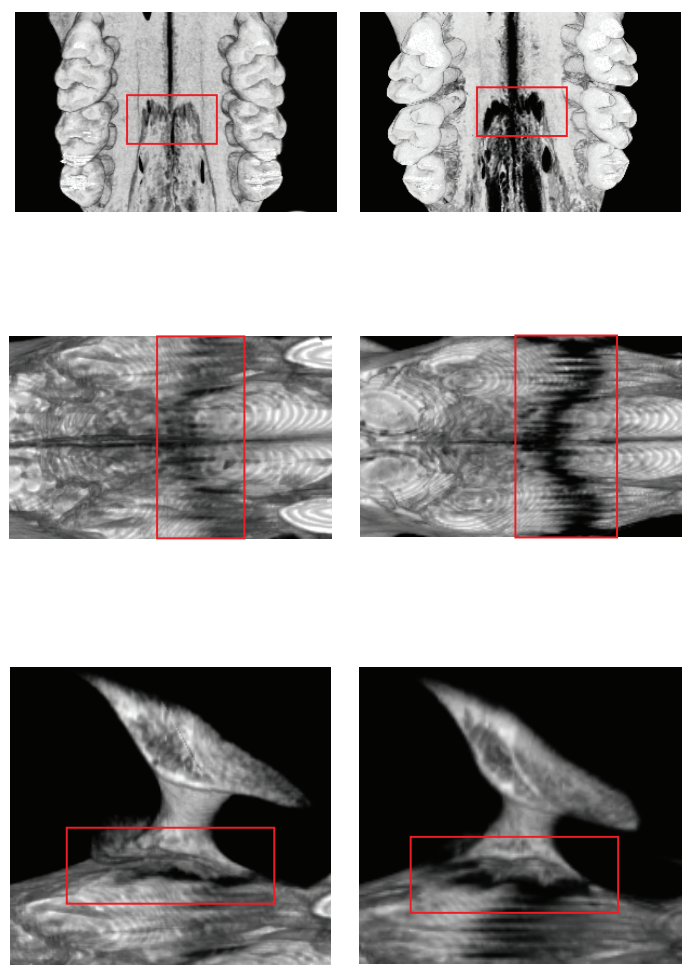

C
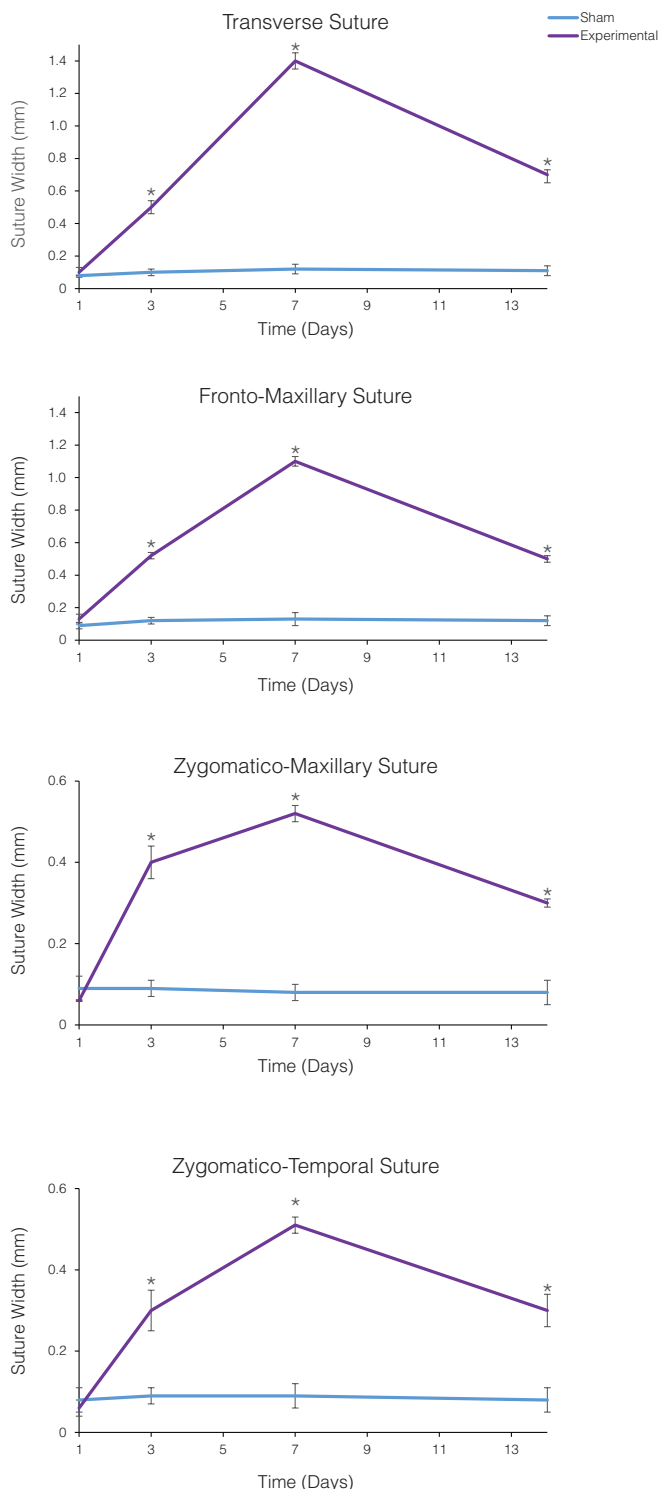

Figure 3: Increase in sutural width in response to transverse forces occurs in both tensile and compressive sutures. (A) 3D $\mu C T$ reconstructed images of rat heads showing surrounding sutures that connect the maxilla to adjacent bones. Arrows point to the location of the zygomaticomaxillary, zygomaticotemporal, transverse and frontomaxillary sutures. (B) 3D $\mu C T$ reconstructed images of the sutures at day 7 for both Sham and Experimental group maxillae show increased width for all the sutures (red box detail). (C) Widths of sutures were measured over time in both Experimental and Sham maxillae. Data expressed as the mean \pm SD of distances in mm. Each number represents the average of 5 samples. ( ${ }^{\star}$ Experimental width significantly different from Sham, $\left.p<0.05\right)$. 
A

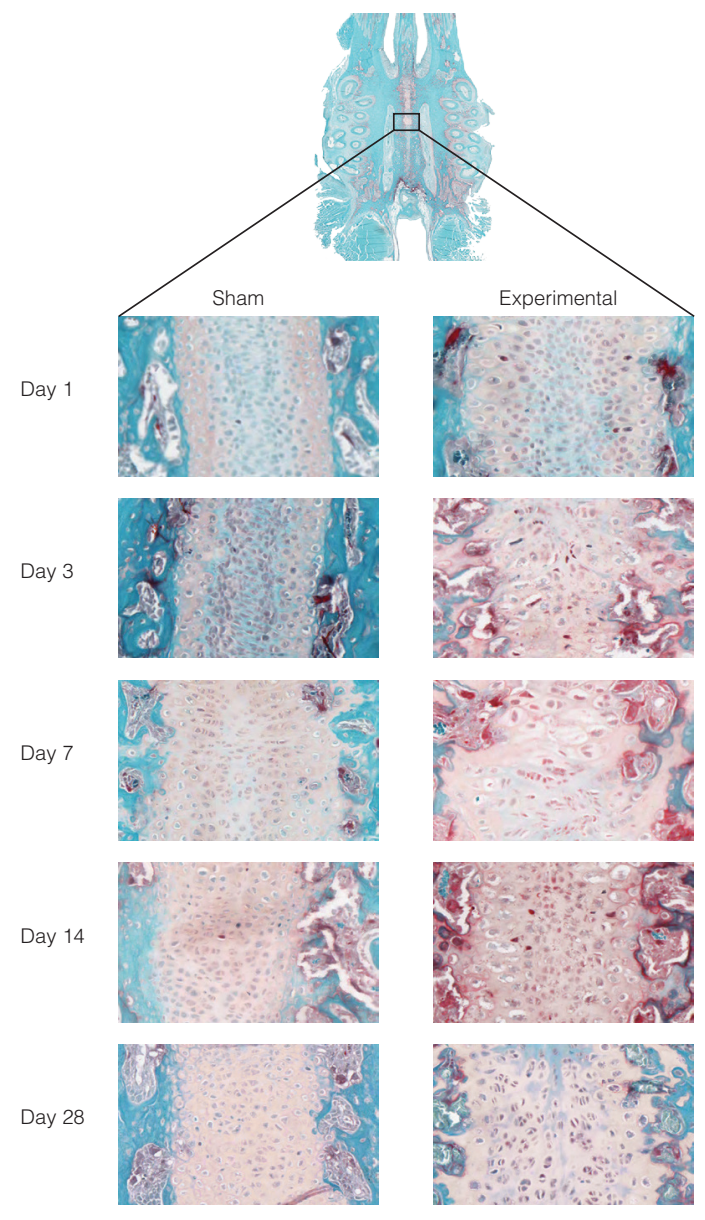

$\mathrm{B}$

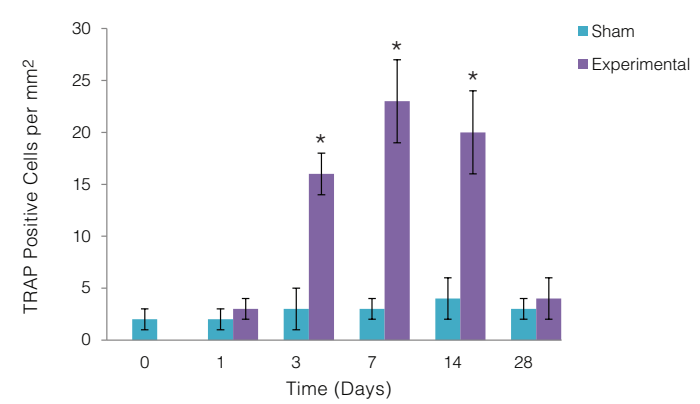

C
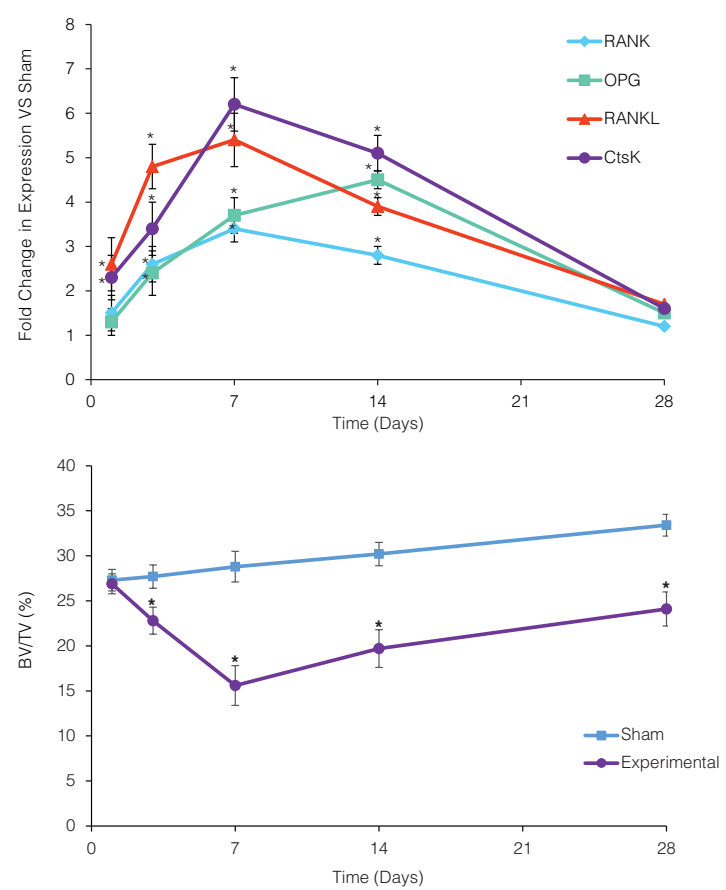

Figure 4. Osteoclast number and activity increase significantly in the mid-palatal suture in response to tensile forces. (A) Immunohistochemistry for TRAP was performed in paraffin section of both Experimental and Sham maxillae to identify active osteoclasts in the area. Light microphotographs show TRAP-positive osteoclasts in the mid-palatal suture and surrounding bone at different time points. All images were collected at the suture area between the first and second molars. Osteoclasts are stained as multi-nucleated red cells (black arrows, magnification 10X). (B) Mean numbers of osteoclasts at different time points, in the area of the mid-palatal suture and adjacent bone. Each value represents the mean $\pm S D$ of five animals. ( ${ }^{*}$ Significantly different from Sham, $\left.p<0.05\right)$. C) Change in expression of osteoclast markers (RANKL, RANK, OPG and CtsK) in the mid-palatal suture of the Experimental and Sham groups at different time points during application of transverse forces was measured by RT-PCR. Data presented as mean "fold" change in expression in comparison to Sham expression. Each number represents the average of 5 samples. (* Significantly different from Sham at the same time point, $p<0.05)$. (D) Bone density was quantified using $\mu C T$ data in the area of the mid-palatal suture and surrounding palatal bone. Data shown as mean \pm SD of BV/TV percentage (bone volume/ tissue volume). ( ${ }^{*}$ Significantly different from Sham at same time point, $\left.p<0.05\right)$.

suture of Experimental animals compared to Sham animals.

To quantify the effect of osteoclast activation, bone density was measured in the palate (Fig. 4D). As expected, the increase in number and activity of osteoclast at the mid-palatal suture and surrounding bone resulted in a significant decreased in the $\mathrm{BV} / \mathrm{TV}$ in the palate of Experimental animals when compared to Sham animals from Days 3-28 $(\mathrm{p}<0.05)$.

\section{Increased and sustained expression of inflammatory markers in both the mid-palatal suture and PDL correlate with osteoclast activity}

Having demonstrated that transverse force produced cellular changes in the mid-palatal suture, including an increase in osteoclast number and activation, we hypothesized that the local environment in the mid-palatal suture immediately after force application would promote osteoclastogenesis through inflammatory mediators, similar to the PDL after orthodontic force application. Expression of 29 inflammatory markers that were previously shown to increase in PDL in response to Orthodontic forces (22) were measured in both the mid-palatal suture and PDL 24 hours after application of transverse orthopedic forces to the rat maxilla. We included the PDL in these experiments for two reasons: 1) as an established model of tissue response to force application and 2) to determine if the inflammatory responses in the PDL and mid-palatal suture are similar following transverse force application.

Comparing Sham and Experimental groups, we found that expression of all 29 inflammatory markers significantly 
A

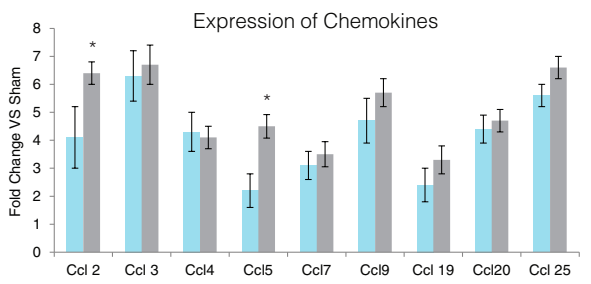

B
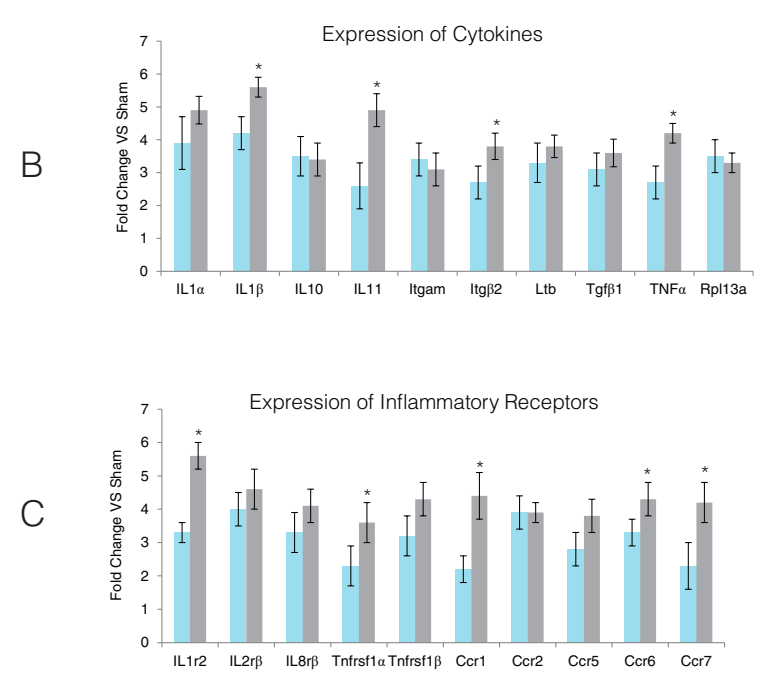
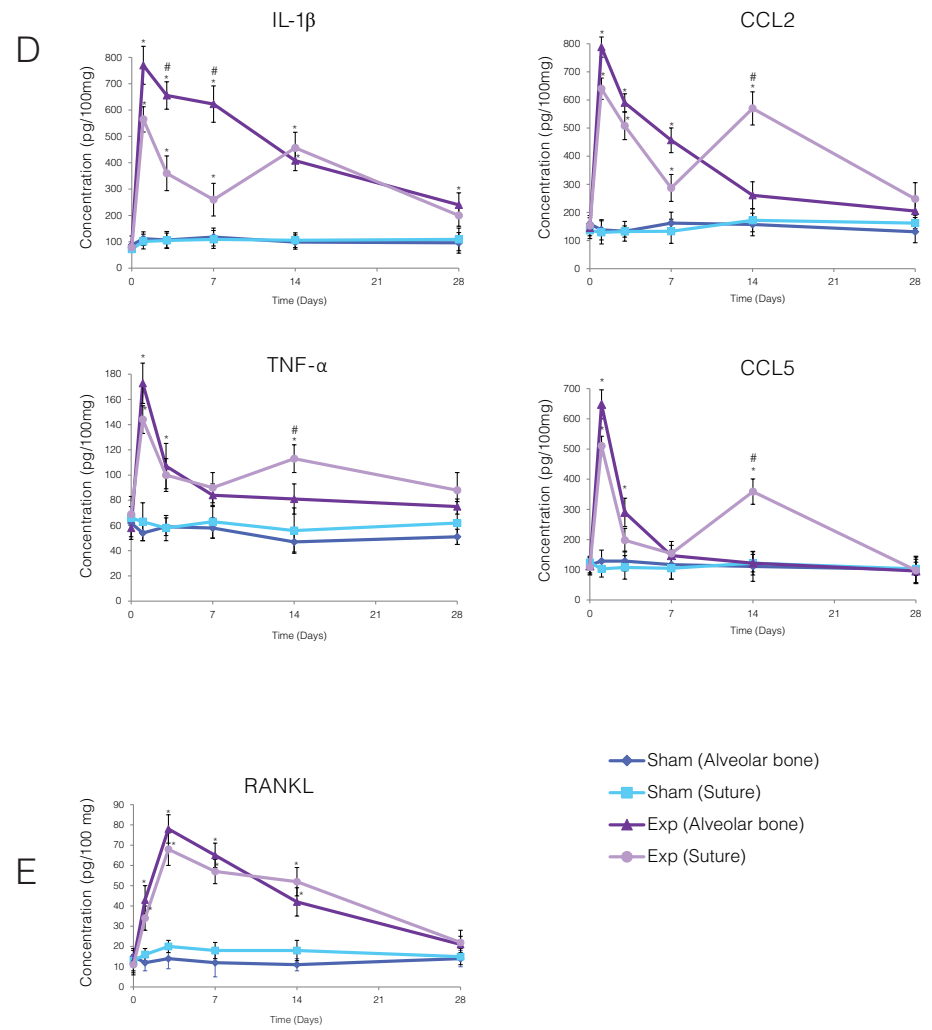

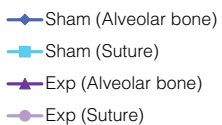

$\rightarrow-\operatorname{Exp}($ Suture)

Suture PDL

Figure 5: Increased and sustained expression of inflammatory markers in both the mid-palatal suture and PDL correlate with osteoclast activity. Expression of chemokines (A), cytokines $(B)$, and their receptors $(C)$ in the mid-palatal suture and PDL of the Sham and Experimental groups was measure by RT-PCR 24 hours after force application. Data are expressed as the Experimental mean "fold" change $\pm S D$ in gene expression compared to the Sham group. Each number represents the average of 5 samples. All 29 genes were significantly different between Experimental and Sham at both locations. ( ${ }^{*}$ Significantly different between suture and PDL in experimental group, $p<0.05$ ). (D) Mean concentration of IL-1 $\beta$, TNF- $\alpha, C C L 2$, CCL5 in mid-palatal suture and PDL at different time points was evaluated by ELISA. Data expressed as the mean $\pm S D$ of concentration in picograms per $100 \mathrm{mg}$ of tissue. Each number represents the average of 5 samples. ( ${ }^{*}$ Significantly different from Sham; \# significantly different between Experimental suture and Experimental PDL, $p<0.05$ ). (E) Mean concentration of RANKL in the mid-palatal suture and PDL of Sham and Experimental groups at different time points was evaluated by ELISA. Data expressed as the mean \pm SD of concentration in picograms per $100 \mathrm{mg}$ of tissue. Each number represents the average of 5 samples. ( ${ }^{\star}$ Significantly different from Sham, $\left.p<0.05\right)$.

increased $(\mathrm{p}<0.05)$ in both the PDL and mid-palatal suture 24 hours after transverse force application (Figs. 5A, 5B and 5C). In the PDL of the Experimental group chemokines increased 3.3- to 6.7-fold, cytokines increased 3.1- to 5.6-fold and cytokine receptors increased 3.6- to 5.6-fold compared to the Sham group. In the mid-palatal suture of the Experimental group, chemokines increased 2.2- to 6.3-fold, cytokines increased 2.2- to 4-fold, and inflammatory marker receptors increased 2.7- to 4.2-fold compared to the Sham group. No significant differences were observed between Sham and Control (no appliance) groups for expression of inflammatory markers in either the PDL or the mid-palatal suture ( $p>0.05$ ) (data not shown).

Interestingly, we also observed significant differences in expression levels of a subset of inflammatory markers in the PDL and mid-palatal suture. Importantly, the differences were consistently higher in the PDL compared to the mid-palatal suture. The chemokines CCL2 and CCL5, the cytokines IL-1 $\beta$ IL-11, Itg $\beta 2$ and TNF- $\alpha$, and the cytokine receptors IL-1r2, Tnfrsf1 $\beta$, CCR, CCR6 and CCR7 were significantly higher in the PDL compared to the mid-palatal suture in the Experimental group $(\mathrm{p}<0.05)$.

To determine if the effect of transverse forces on inflammatory marker expression was transient or sustained, the levels of the cytokines IL-1 $\beta$ and TNF- $\alpha$ and chemokines CCL-2 and CCL-5 in the mid-palatal suture and PDL were followed for 28 days after application of transverse force (Fig. 5D). In general, time course of the expression of all 4 cytokines and chemokines differed in the mid-palatal suture and PDL. Levels of each cytokine and chemokine peaked twice in the mid-palatal suture, once on Day 1 and again on Day 14. This contrasts with the expression pattern in the PDL, where each cytokine and chemokine peaked only on Day 1. While 3 of the 4 genes retuned to baseline in both the mid-palatal suture and PDL by Day 28 , IL- $1 \beta$ remained significantly elevated in the PDL throughout the time course.

The cytokines and chemokines we assayed have an important role in osteoclast recruitment and activation in the PDL in response to orthodontic forces $(22,23)$. To further confirm 


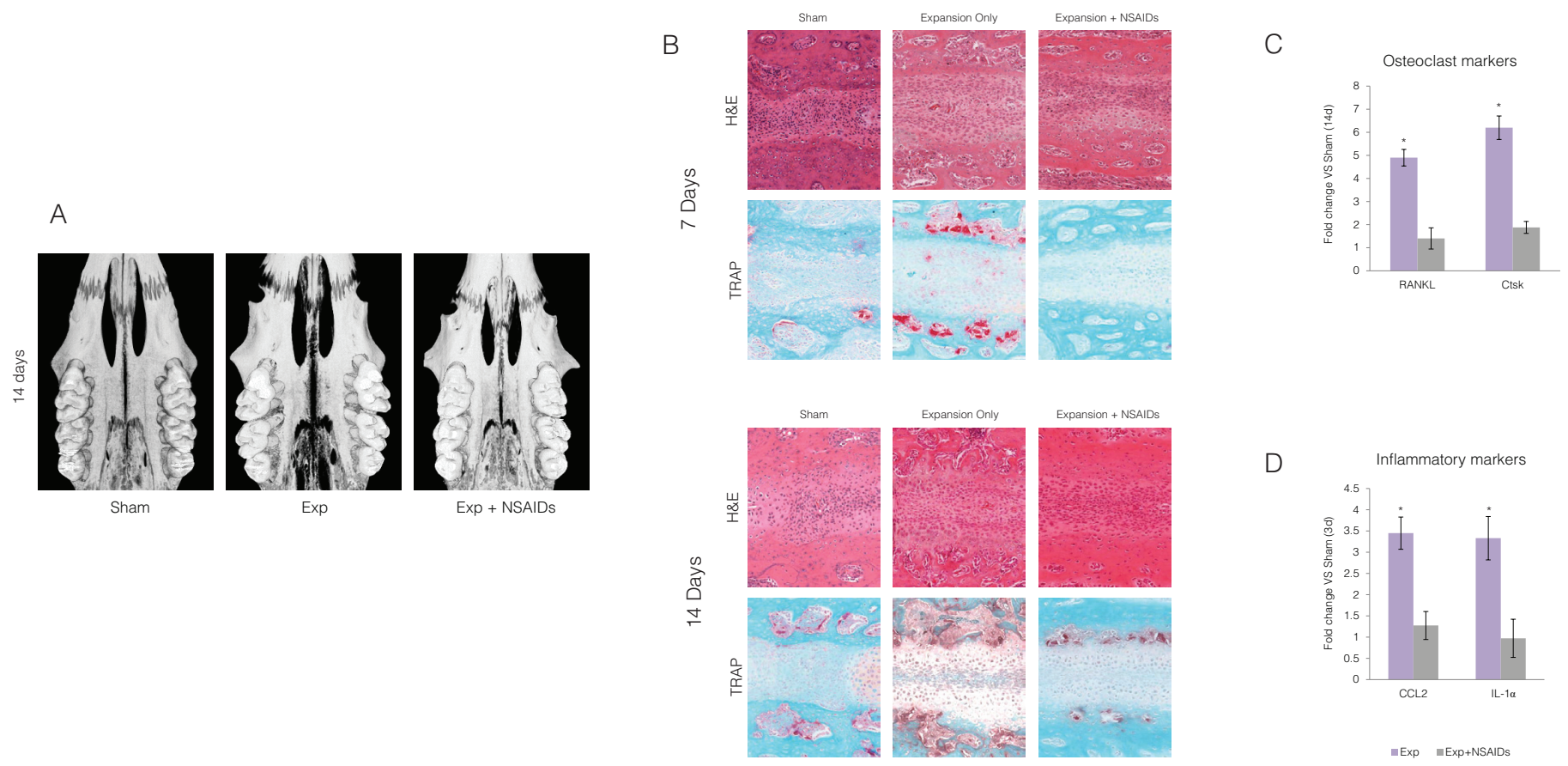

Figure 6: Anti-inflammatory medication blocks the catabolic effect of transverse forces. (A) $\mu C T$ images of representative maxillae from Sham, Experimental, and Experimental + NSAIDs groups after 14 days of force application. (B) H\&E and TRAP staining of histological sections taken from the mid-palatal suture in the area of contact between the first and second molars in Sham, Experimental and Experimental + NSAIDs groups after 7 and 14 days of transverse force application. Osteoclasts are red multinucleated cells (black arrows). Change in expression of (C) RANKL, Ctsk and (D) IL-1 $\beta$ and CCL2 in the mid-palatal suture in the presence and absence of NSAID was measured by RT-PCR at Days 3 and 7. Data are expressed as mean \pm SD "fold-change" in expression in comparison to Sham. Each value represents the average of 5 samples. ( ${ }^{*}$ Significantly different from Sham group, $\left.p<0.05\right)$.

\begin{tabular}{lcccccc} 
& \multicolumn{6}{c}{ Days } \\
\cline { 2 - 7 } & 0 & 1 & 3 & 7 & 14 & 28 \\
Palatal Width & & & & & & \\
Sham & $1.89 \pm 0.15$ & $1.89 \pm 0.15$ & $1.99 \pm 0.17$ & $2.06 \pm 0.16$ & $2.33 \pm 0.15$ & $2.73 \pm 0.17$ \\
Exp & & $1.92 \pm 0.22$ & $2.08 \pm 0.24$ & $2.22 \pm 0.24$ & $2.87 \pm 0.28^{\star \#}$ & $3.65 \pm 0.36^{\star \#}$ \\
Exp + NSAID & & $1.91 \pm 0.23$ & $2.03 \pm 0.23$ & $2.12 \pm 0.24$ & $2.31 \pm 0.27$ & $2.91 \pm 0.25$
\end{tabular}

Dental Width

\begin{tabular}{|c|c|c|c|c|c|c|}
\hline Sham & $3.12 \pm 0.16$ & $3.12 \pm 0.21$ & $3.84 \pm 0.22$ & $4.24 \pm 0.24$ & $4.3 \pm 0.22$ & $4.42 \pm 0.24$ \\
\hline Exp & & $3.18 \pm 0.22$ & $3.97 \pm 0.25$ & $4.43 \pm 0.39$ & $5.19 \pm 0.42^{\star \#}$ & $5.68 \pm 0.51^{\text {*\# }}$ \\
\hline Exp + NSAID & & $3.15 \pm 0.22$ & $3.89 \pm 0.24$ & $4.28 \pm 0.25$ & $4.27 \pm 0.47$ & $4.69 \pm 0.44$ \\
\hline
\end{tabular}

\begin{tabular}{|c|c|c|c|c|c|c|}
\hline Sham & $0.08 \pm 0.02$ & $0.08 \pm 0.02$ & $0.10 \pm 0.03$ & $0.11 \pm 0.04$ & $0.1 \pm 0.03$ & $0.1 \pm 0.03$ \\
\hline Exp & & $0.11 \pm 0.02$ & $0.53 \pm 0.04^{*}$ & $1.2 \pm 0.03^{\star}$ & $0.64 \pm 0.02^{\star \#}$ & $0.29 \pm 0.04^{\star \#}$ \\
\hline Exp + NSAID & & $0.07 \pm 0.04$ & $0.11 \pm 0.04$ & $0.12 \pm 0.03$ & $0.12 \pm 0.04$ & $0.11 \pm 0.04$ \\
\hline
\end{tabular}

TABLE 1: Morphological changes in the maxillary palatal, dental and sutural widths in response to application of transverse forces in the absence (Exp) and presence (Exp + NSAID) of anti-inflammatory medication. Data shown as mean + SEM of 5 samples ( ${ }^{*}$ Significantly different from Sham; \# Significantly different from Exp +NSAID) 
that the elevation of these cytokines correlated with osteoclastogenesis in both the suture and PDL, ELISA was performed for the osteoclast marker RANKL from protein extracts from the mid-palatal suture and PDL and surrounding alveolar bone, at different time points (Fig. 5E). RANKL protein in the mid-palatal suture was significantly higher at Days 1, 3, 7 and $14(\mathrm{p}<0.05)$ in the Experimental group compared to the Sham group (2.1-, 3.4-, 3.1-, 2.8-fold, respectively); however, no difference was observed at Day 28. Similarly, RANKL protein level increased in the PDL of the Experimental groups compared to the Sham group on Days 1, 3, 7 and 14 (3.5-, 5.6-, 5.4- and 3.8-fold, respectively) $(\mathrm{p}<0.05)$, while no difference was observed on Day 28 ( $p>0.05)$.

A

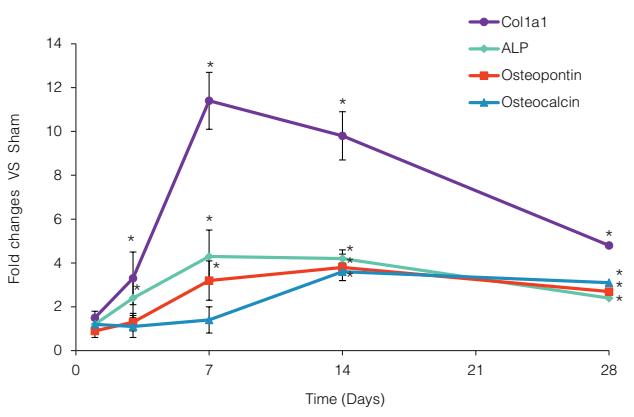

B

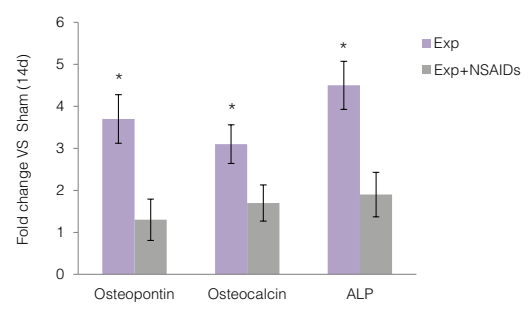

C

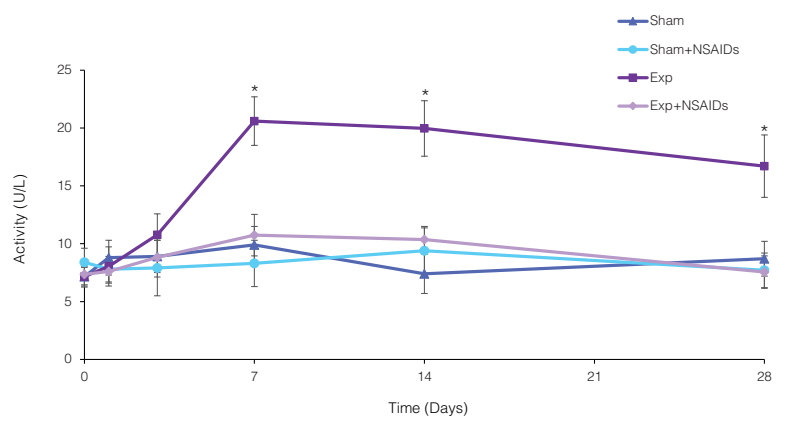

Inflammation and osteoclast activation were prerequisites for skeletal changes in response to transverse forces

To evaluate how inhibiting inflammation and osteoclast activity with NSAIDs affects the skeletal response to transverse forces, $\mu \mathrm{CT}$ images of Sham, Experimental and Experimental + NSAID groups were evaluated. Daily records of weight and general health of the animals confirmed that NSAID treatment did not cause any systemic effect during the 28 days of the study (data not shown). Measurements of $\mu \mathrm{CT}$ images of maxillae from Sham, Experimental and Experimental +NSAID groups demonstrated no increase in either palatal and dental expansion after applying transverse forces in the presence of NSAID (Fig. 6A and Table 1). Differences in palatal and dental widths between Experimental and Experimental + NSAID groups were statistically significant $(\mathrm{p}<0.05)$ (Table 1$)$.

$\mathrm{D}$

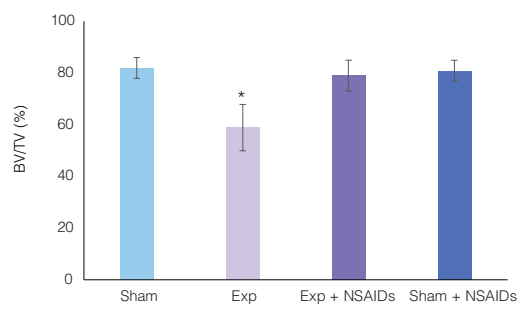

E
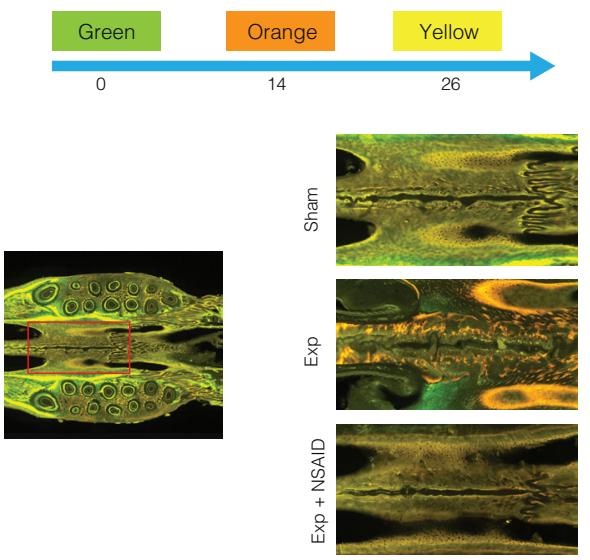

Figure 7: Osteoclasts activity is a pre-requisite for osteoblast activity in the mid-palatal suture in response to transverse forces. (A) Change in expression of osteogenic markers (collagen I, ALP, Osteopontin and osteocalcin) in the mid-palatal suture at different time points by RT-PCR. Data are expressed as mean \pm SD "fold-change" in expression in comparison to Sham. Each value represents the average of 5 samples. ( ${ }^{*}$ Significantly different from Sham group, $\left.p<0.05\right)$. (B) Change in expression of $A L P$, Osteopontin and Osteocalcin in the mid-palatal suture in the presence or absence of anti-inflammatory medication was measure by RT-PCR at Day 14. Data are expressed as the mean $\pm S D$ "fold-change" in expression compared to Sham. Each value represents the average of 5 samples. $\left({ }^{*}\right.$ Significantly different from Experimental + NSAID group and from the Sham group, $p<0.05$ ). (C) Mean activity of ALP during 28 days was measure spectrophotometrically. Transverse forces were able to increase $A L P$ in Experimental group. Each value represents the average of 5 samples. ( ${ }^{*}$ Significantly different from Sham group, $\left.p<0.05\right)$. (D) Bone density was quantified using $\mu C T$ data in the inter-radicular area of the first maxillary molar at Day 28. Data shown as mean \pm SD of BV/TV percentage (bone volume/ tissue volume) $\left({ }^{*}\right.$ Significantly different from Sham group, $p<0.05)$. (E) Fluorescence microscopy images of mid-palatal suture after 28 days of expansion. Bone labeling was performed by Calcein on days 0 (Green), Xylenol Orange at day 12 (Orange) and Demoxicycline at day 26 (Yellow). Experimental group maxilla demonstrates significant bone formation activity around the suture evidenced by increase fluorescence, which was reduced in animals that received anti-inflammatory medication (Experimental +NSAIDs). 
To further evaluate how anti-inflammatory medication affects osteoclast number in the mid-palatal suture, H\&E and TRAP staining were performed in sections from the mid-palatal suture of Sham, Experimental and Experimental + NSAID groups (Fig. 6B). On Days 7 and 14, transverse forces in the Experimental group resulted in suture widening and marked resorption of the suture's bony borders. TRAP staining in the Experimental group revealed a significant increase in the number of osteoclasts at both Day 7 and Day 14. However, in the Experimental + NSAID group, no widening or bone resorption was observed. TRAP staining in the Experimental + NSAID group was significantly reduced on Days 7 and 14 . To further confirm the reduction in osteoclastic activity in the suture, the expression of RANKL and Cathepsin K (CtsK) was measured in the suture seven days after applying transverse forces (Fig. 6C). Anti-inflammatory medication decreased RANKL expression significantly from 4.7-fold in the Experimental group to 1.4-fold in the Experimental + NSAID group $(\mathrm{p}<0.05)$. Similarly, CtsK expression decreased significantly from 5.8to 1.6-fold in response to the medication $(\mathrm{p}<0.05)$.

To verify that NSAID treatment affected osteoclast formation and skeletal changes through inhibition of inflammatory markers, expression of prominent inflammatory markers CCL2 and IL-1 $\beta$ were measured in the mid-palatal suture of different groups three days after applying transverse forces (Fig. 6D). Expression of CCL2 in the Experimental group

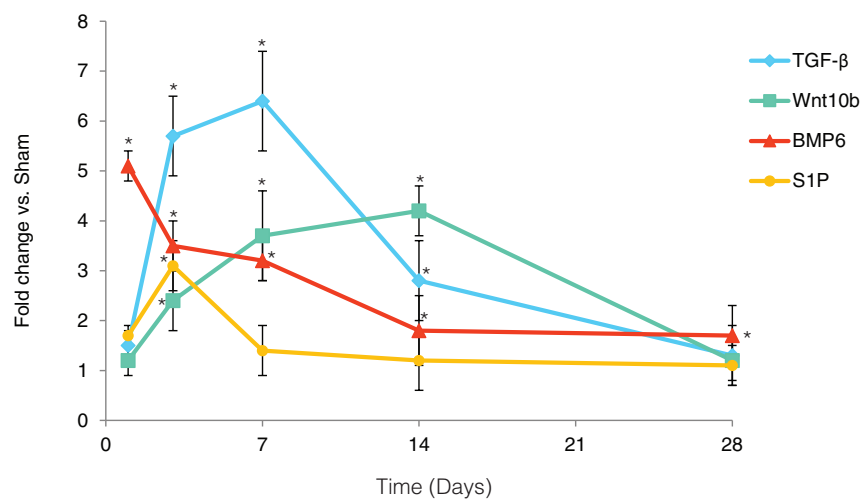

Figure 8: Expression of transitional molecules activated during sutural response to transverse forces. Gene expression of BMP-6, TGF- $\beta$ and Wnt10b in the mid-palatal suture was measured by RT-PCR from Day 3 to Day 28. Data expressed as the mean $\pm S D$ "fold" change in comparison to Sham group. Each value represents the average of 5 samples ( ${ }^{*}$ significantly different from Sham group, $p<0.05$ ).

increased 3.6-fold compared to the Sham group. However, this increase was blocked with NSAID administration (1.3-fold increase compared to the Sham group). IL-1 $\beta$ expression in the Experimental group increased 3.2-fold compared to the Sham group. As with CCL2, the same force in the presence of medication (Experimental + NSAID) did not stimulate expression of IL-1 $\beta$ in the suture (1.1-fold change compared to the Sham group) (Fig. 6D). The decrease in IL-1 $\beta$ and CCL2 expression in response to NSAID was statistically significant $(p<0.05)$.

\section{Bone formation at the mid-palatal suture was a delayed reaction to transverse forces and required osteoclast activation.}

Since we demonstrated that transverse force triggered osteoclast activity in the circum-maxillary sutures, we next investigated whether the osteogenic activity was also affected. We examined the time course of expression of the osteogenic markers Colla1, ALP, osteopontin and osteonectin at Days 1, 3, 7, 14, and 28 after applying transverse force (Fig. 7A). Colla1 and ALP levels increased significantly in the Experimental group at Day 3 (3.3- and 2.4-fold, respectively), peaked at Day 7 (11.4- and 4.3-fold, respectively) and stayed elevated at Days 14 (9.8- and 4.2-fold, respectively) and 28 (4.8- and 2.4-fold, respectively), compared to the Sham group $(\mathrm{p}<0.05)$. Osteopontin increased significantly at Days 7, 14 and 28 (3.2-, 3.8- and 2.7-fold, respectively) compared to the Sham group $(\mathrm{p}<0.05)$, while osteocalcin levels peaked at Day 14 with a significant increase (3.4-fold) compared to the Sham group and stayed elevated until Day 28 (3.1-fold increase) $(\mathrm{p}<0.05)$ (Fig. 7A).

When we examined the expression of osteoblasts markers 14 days after expansion in the presence of low dose anti-inflammatory medication, a sharp decrease in expression levels was observed. Expression of osteogenic markers ALP, osteopontin, and osteocalcin in the Experimental + NSAID group was significantly lower (1.9-, 1.4-, and 1.2-fold, respectively) compared to the Experimental group, but was not significantly different than the levels in the Sham group $(\mathrm{p}<0.05)$ (Fig. 7B).

To investigate if the low doses of NSAID used in this study directly affect the osteoblast activity, levels of ALP were measured in the Sham, Experimental, Experimental + NSAIDs, and a Sham group that received NSAIDs but no transverse forces (Fig. 7C). No change in baseline ALP level was observed between the Sham and Sham + NSAID groups, while the Experimental group showed a statistically significantly higher level of ALP on Days 7, 14 and 28, with peak ALP levels at Day $7(p<0.05)$. There was no statistical difference in ALP activity between Experimental + NSAID and Sham groups, which demonstrates that this low NSAID dose did not have a systemic effect on the baseline activity of osteoblasts. Furthermore, when we evaluated bone density in the inter-radicular area of the first maxillary molar at Day 28 (Fig. 7D), we found a significant decreased in the BV/TV in the Experimental animals compared to Sham animals $(\mathrm{p}<0.05)$. No statistical difference between Experimental + NSAID, Sham + NSAID, and Sham groups was observed, which demonstrates the drug also did not have any significant effect on osteoblasts activity in response to functional forces.

To further evaluate the effect of anti-inflammatory medication on overall bone formation during 28 days of palatal expansion, bone activity around the mid-palatal suture was compared between the Sham, Experimental and Experimental + NSAID groups using fluorescent dye uptake and fluorescence microscopy. While the Sham group showed only basal activity 
in one month, the Experimental group demonstrated a significant increase in bone formation revealed by green, orange, and yellow fluorescence. The Experimental + NSAID group did not show an increase in fluorescence activity (Fig. 7E). Based on these results, it is clear that a possible direct effect of NSAIDs on osteoblasts cannot explain the significant decrease in osteoblast activity in the sutures in response to transverse forces.

\section{Stimulating bone formation helped to transition from the catabolic phase to the anabolic phase.}

To further study the relation between osteoclasts and osteoblasts, we investigated the expression of different molecules that may be released in the expansion area, directly or indirectly, in response to osteoclast activity. It has been suggested (24-26) that osteoclasts may activate osteoblasts through activation of TGF- $\beta$, BMP6, WNT10b and SP1. Therefore, the change in expression of these markers in the mid-palatal suture was studied at Days 1, 3, 7, 14, and 28 after the start of transverse force application and were compared to the Sham group (Fig. 8). TGF- $\beta$ expression was highest at Days 3 and 7 and returned to baseline by Day 28. BMP6 peaked at Day 1 and dropped throughout the remainder of the study. WNT10b levels steadily increased until they peaked at Day 14 and dropped to baseline by Day 28. SP1 levels peaked at Day 3 and returned to baseline on Days 7-28. All of these increases were statistically significant compared to the Sham group $(\mathrm{p}<0.05)$.

\section{Discussion}

\section{Sutural opening in response to transverse forces is a biological phenomenon}

Application of transverse force to the maxilla have been traditionally advocated for orthopedic correction in patients with maxillary constriction $(27,28)$. There are two general assumptions behind these treatments: 1) heavy forces separate the two hemi-maxillae creating tensile stress in the mid-palatal suture, and 2) tensile stress has direct osteogenic effect on the mid-palatal suture (29-33). Based on the first assumption, opening of the mid-palatal suture has been considered to result directly from hemi-maxillae displacement in response to transverse forces, making expansion a physical, rather than a cellular, phenomenon. This concept is so predominant in Orthodontics that the majority of research in this field has focused on stress and strain distribution using dry skulls or finite element analysis $(34,35)$. These studies try to predict maxillary movement in response to transverse forces based on the distribution of stresses (36). However, if a biological response is not only a part of the process, but it is the main part of the skeletal response to transverse forces, then these predictions misrepresent the clinical reality, which could explain the conflicting results between these in vitro studies and clinical studies (37-39).

We were surprised by our findings that physical displacement of the hemi-maxillae was not the first event in response to transverse forces. Instead, suture widening occurred before skeletal changes. Our experiments demonstrate that while there was an immediate skeletal and dental displacement in response to continuous transvers forces, these movements were minuscule and halted quickly. In fact, the first significant increase in palatal and dental width did not occur until a few days after maximum sutural widening. In addition, the magnitude of increase in the mid-palatal suture width was not linearly related to the magnitude of skeletal displacement, which argues that sutural opening cannot be explained solely by physical displacement of hemi-maxillae. These conflicting results contradict the general understanding that expansion is an immediate response to applied force and suture widening is the direct result of skeletal displacement. Our histological studies confirmed these findings by demonstrating the appearance of TRAP+ osteoclasts in sutures in the early days of transverse forces application, which coincided with suture opening ahead of skeletal changes as seen in the $\mu \mathrm{CT}$ images. Correlated to the increase in osteoclast activity, BV/TV in the bone surrounding the mid-palatal suture significantly decreased. From this aspect, maxillary expansion is very similar to tooth movement, where movement does not occur until osteoclasts have been activated ahead of movement to resorb the bone in the path of movement.

In support of our conclusion that a catabolic phase is an important part of skeletal changes in response to tensile forces, our results demonstrate that application of anti-inflammatory medication significantly decreased the skeletal changes in response to transverse forces, emphasizing the requirement for osteoclasts in the skeletal changes. If these changes were just a physical phenomenon, one would not expect the exposure to anti-inflammatory medication to significantly affect skeletal changes.

Our findings are also in agreement with previous observations that incremental application of high-magnitude transverse forces in a dry skull (where there is no biological reaction) rapidly causes stress points to appear at points where the maxilla abuts adjacent bones. If the transverse force is continuously applied, these stress points do not disappear and, may ultimately cause fractures (40). On the other hand, stresses generated in vivo in response to incremental forces dissipate gradually (if there is enough time between force application) (31) and prevent further damage to adjacent bones. Indeed, the rapid delivery of high-magnitude transverse force that bypasses this biological prerequisite for expansion can cause fracture of adjacent skeletal structure (41), presenting a significant danger to the patient. These studies emphasize the vital role of the biological reaction in response to transverse forces not only as a necessary step before physical maxillary expansion, but also as a natural safety mechanism to prevent stress build up around vital organs.

\section{Catabolic response in the suture is independent of the type of stress applied to the suture}

What was surprising about our data was that the catabolic 
effects were stimulated in response to transverse forces. The fact that transverse force (tensile stress) stimulated resorption argues against the current belief that only compressive forces (compressive stress) are catabolic (42). The immediate catabolic response was not only observed in the mid-palatal suture, but in all circum-maxillary sutures. This is very interesting since it has been shown that different types of stresses appear in the circum-maxillary sutures in response to transverse forces $(35,36,43)$. The fact that in all of these sutures, the catabolic reaction was the immediate response, suggests that the microtrauma induced by different types of stress is the main factor in stimulating a catabolic reaction.

This general increase in width of the circum-maxillary sutures ahead of large mid-palatal transverse changes may provide the required mobility for the hemi-maxillae and space for subsequent transverse movement. The increase in the mid-palatal suture width mostly followed the width increases in the zygomaticomaxillary, zygomaticotemporal, and frontomaxillary sutures, which are all directly in the path of the forces generated by maxillary expansion. One would expect that in the absence of this widening, the skeletal changes will be impossible, as was observed in animals that were exposed to anti-inflammatory medication. Based on these observations, our data support the conclusion that the zygomatic bone - not the mid-palatal suture - is the main resistance against maxillary opening (44). This can also explain why previous studies did not report any facilitated expansion in cleft patients, where the mid-palatal suture is completely or partially missing (45-47).

\section{Inflammatory markers and osteoclast activators are medi- ators of transverse force response in the maxilla}

Since osteoclastogenesis is largely regulated by inflammatory markers, we investigated whether transverse maxillary forces increase osteoclasts through activation of inflammatory markers. Many of the inflammatory cytokines, chemokines and their receptors significantly increased in the mid-palatal suture 24 hours after transverse force application. Our data further shows that expression of inflammatory markers in the suture coincide with increased expression of osteoclast markers such as RANK and RANKL (48-50), which signal the start of catabolic changes at the suture. The fact that blocking the inflammatory markers with anti-inflammatory medication inhibited expression of osteoclast activators strongly supports the role of inflammation in osteoclastogenesis and initiation of bone resorption, in agreement with previous reports (51-54).

Since the appliance used in our experimental model was tooth-borne, transverse forces not only stimulated inflammatory reactions in the sutures but also in the PDL. Interestingly, the profile of inflammatory markers was similar in the suture and PDL, which emphasizes that the inflammatory response is largely a general response to trauma and is not tissue-specific. This traumatizing effect and subsequent inflammatory reaction can explain the pain and discomfort the patient experiences in the surrounding sutures if excessive force is applied.
In our experimental model, two differences in the inflammatory marker profiles of the mid-palatal suture and the PDL appeared. First, the PDL showed a stronger inflammatory reaction compared to the suture. One can argue that based on anatomical variations between the suture and the PDL, it is easier to induce an inflammatory reaction, and reach the saturation point for inflammation, in the PDL than in the suture (22). Compared to the mid-palatal suture, the PDL is much thinner than the suture and has a relatively richer blood supply that will allow for a more rapid inflammatory response compared to the suture. Second, when we assayed four inflammatory markers over the course of the experiment, each demonstrated two peaks of activation in the suture but only one peak in the PDL. The exact mechanism for this response is unknown. It is possible that the first peak in both the suture and PDL can be related to the immediate micro-trauma induced by forces, while the second peak in the mid-palatal suture may result from additional stress arising from the complex skeletal movements following the circum-maxillary sutural widening. We did observe that the second peak of inflammatory markers appeared to coincide with the skeletal movement, which may expose the mid-palatal suture to further micro-trauma for a second time. Further research into this phenomenon is required.

Based on these data, the additional increase in the dental width compared to skeletal width can be due to the additive effect of tooth movement in the alveolar bone (as expression of inflammatory makers in the PDL suggest) plus the displacement of the hemi-maxillae that carry the teeth buccally. In addition, dental tipping and alveolar bending due to the distance between the point of force application on the teeth and their center of resistance should not be ignored as contributors to the dental expansion we measured $(55,56)$.

\section{Molecular and cellular transitions drive the skeletal response to tensile force from the catabolic phase to the anabolic phase}

As we discussed earlier it has been assumed that tensile stress directly stimulates bone formation. But, is that true? One would expect that if osteogenesis was the direct result of tensile stress it should start immediately upon force application. To our surprise, markers of the anabolic (osteogenic) phase were not detected until the middle of the catabolic phase, which was activated immediately after force application. Based on this observation one would wonder if the catabolic phase is a prerequisite for the anabolic phase. Our results demonstrate that in the presence of anti-inflammatory medication, when the catabolic effect was decreased significantly, the expression of osteogenic markers was also significantly reduced. Moreover, bone formation detected with immunofluorescence was significantly blocked by NSAID treatment. One could argue that the decrease in bone formation activity was due to the direct effect of anti-inflammatory medication on osteoblasts. Research in this area is not clear. While some reported inhibitory effects of high dose anti-inflammatory medication on osteoblasts, others 


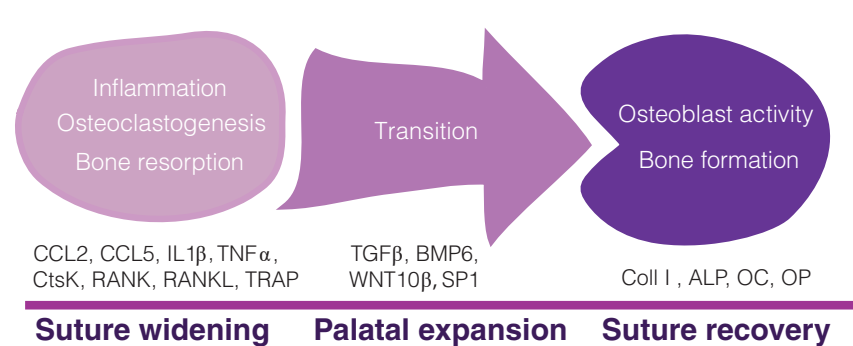

Figure 9: Model of events in sutures in response to orthopedic transverse tensile forces. As an initial response to tensile forces, a robust and sustained inflammatory response (markers: IL1- $\beta$, TNF- $\alpha, C C L-2, C C L-5$ ) recruits osteoclasts precursors into the area of mid-palatal suture and adjacent bone. As a result of osteoclast activity (markers: RANK and RANKL, CtsK, TRAP) and bone resorption, there is a significant reduction in the bone density in the area and the suture width is visibly increased. After this initial stage of overt catabolism in both tensile and compressive sutures, there is a transition marked by stimulation of bone formation via coupling factors including diffusible factors, membrane bound molecules and factors embedded in bone matrix (markers: TGF- $\beta, B M P 6$, WNT10b and SP1). During this period and only after the catabolic stage, does the palatal and dental widths significantly increase, and physical expansion finally occurs. This transition period results in osteoblast stimulation and is followed by a strong anabolic phase (markers: Coll I, ALP,OC, OP) that ensures that the resorbed bone is replaced with new bone, re-establishing the integrity of the suture in the framework of the new skeletal dimensions.

have shown that low dose of anti-inflammatory medication, similar to what was used in our experiments, does not affect osteoblast activity (57-59). Our data confirmed that there were no changes in the basal or functional activity of osteoblasts in animals exposed to anti-inflammatory medication. On the other hand, a decrease in osteoclasts number and activity induced by transverse forces in the presence of low dose anti-inflammatory medication coincided with a decrease in osteoblast activity, which suggests a possible role for osteoclasts in the activation of osteoblasts, in agreement with previous studies (24-26).

The final piece of evidence to support our conclusion that the inflammation-dependent catabolic phase must precede the anabolic phase in the biological response to tensile stress is the temporal profile of genes that drive the transition of the bone remodeling machinery from the catabolic to the anabolic phase.

It has been shown that osteoclasts may activate osteoblasts by the release of diffusible paracrine factors such as sphingosine 1-phosphate (S1P), BMP6 and Wnt10b (24). Our experiments show the presence of TGF- $\beta$, S1P, Wnt10b, BMP6 at the suture at the time points coinciding with the transition phase marked by an increase in osteoblastic markers and a decrease in osteoclastic markers. It should be emphasize that there are at least two other modes of osteoclast-osteoblast communication that were not investigated in this study: 1) direct contact between these two cells allowing membrane-bound ligands and receptors to interact and initiate intercellular signaling, and 2) during bone resorption, osteoclasts may liberate molecules deposited in bone matrix such as TGF- $\beta$ or IGF-1 which attract and activate osteoblasts (24-26). Further research in this area is necessary. Therefore, we conclude that the current model of tensile force directly and immediately triggering an osteogenic response is over-simplified and misleading.

\section{Clinical implications of sutural biological response to transverse forces: Osteoclasts as a precision biological knife}

The overall understanding that sutural opening is a cellular reaction rather than a strictly physical phenomenon has a significant impact on clinical decision making regarding the magnitude of transverse forces and rate of force application for patients with maxillary constriction. If, as we have previously believed, separating the hemi-maxillae is a purely physical phenomenon, while tooth movement is a physiological phenomenon, then high forces to overcome sutural resistance is justified, especially in adults. A faster rate of force delivery would achieve skeletal changes before the more time-consuming biological pathways needed for dental changes occur. On the other hand, our data showing that the separation of hemi-maxillae is also a biological phenomenon suggests that the optimum force should be defined based on the optimum biological response, making the use of high forces not only unnecessary but contraindicated as this is known to cause severe damage to the skull (60). Indeed, if the rate of expansion depends on the rate of osteoclast activity, application of higher forces does not increase the rate, but mostly causes accumulation of stress and bending of structures. This hypothesis can explain why previous studies did not show significant differences in the magnitude of expansion between heavy and light forces (41).

Armed with this new understanding of the biological component of maxillary expansion, we can now view the biological response in the suture as a biological knife that will allow clinicians to precisely control maxillary orthopedic movements. In general, the catabolic phase of expansion will define the magnitude of skeletal changes. In addition, if osteoclasts play a significant role in activating osteoblasts, perhaps our treatment should maximize osteoclast activity in the early phase of expansion for improved osteogenic response. Therefore, from a biological standpoint, the clinician should consider osteoclasts the main target of orthopedic treatment.

\section{Conclusion}

We have proven our hypothesis that applying tensile force to the mid-palatal suture stimulates the release of inflammatory markers resulting in osteoclast recruitment and activation, causing widening of the suture. This response is then followed by skeletal and dental widening of the palate and osteoblast activation that re-establishes the bone integrity at the suture. This response is a biological response that mimics the wellknown biological response of orthodontic force on the PDL during tooth movement.

Based on our result, the target of orthopedic treatment by application of static forces is not the bone but the suture. In this regard, any type of stress on the suture can induce microtrauma that will produce a mild inflammatory reaction, which in turn activates osteoclasts in the suture. Activating osteoclasts is a necessary step for the activation of osteoblasts that follows. 
Based on these results, orthopedic treatment in the craniofacial area has two consecutive phases, a catabolic phase defined by sutural opening, and a delayed anabolic phase that reestablishes the sutural width (Fig. 9). The catabolic phase defines the magnitude of the maxillary movement and, therefore, should be optimized to achieve maximum orthopedic correction, while the anabolic phase contributes to the stability and integrity of the skeleton after maxillary displacement. The goal of orthopedic research therefore, should be to determine the optimized force regiment that maximizes a catabolic response during movement and anabolic response during retention. The optimized force regimen for these two phases is not the same, warranting future research.

\section{INNOVATION}

Our findings are novel and support a new theory for the sutural response to orthopedic forces, which emphasizes the importance of osteoclasts in the process and makes these cells the possible target of orthopedic treatment: 1) as the "biological knife" at the sutures that allows the separation of hemi-maxillae for orthopedic movement, and 2) as the trigger for osteoblast activation and bone regeneration when we need to stimulate bone formation, especially at the surface of the cortical bone.

\section{Abbreviations}

NSAID - nonsteroidal anti- inflammatory drugs

$\mu \mathrm{CT}$ - micro computed tomography

RT - PCR - reverse transcriptase polymerase chain reaction PDL - periodontal ligament

\section{Funding}

Funding for this work was provided by Consortium for Translational Orthodontic Research (CTOR), a private research center dedicated to the advancement of Orthodontics and Craniofacial Biology.

\section{References}

1. Honda A, Umemura Y, Nagasawa S. Effect of high-impact and low-repetition training on bones in ovariectomized rats. J Bone Miner Res. 2001;16(9):1688-93.

2. Tanaka M, Toyooka E, Kohno S, Ozawa H, Ejiri S. Long-term changes in trabecular structure of aged rat alveolar bone after ovariectomy. Oral Surg Oral Med Oral Pathol Oral Radiol Endod. 2003;95(4):495-502.

3. Alikhani M, Khoo E, Alyami B, Raptis M, Salgueiro JM, Oliveira SM, et al. Osteogenic effect of high-frequency acceleration on alveolar bone. J Dent Res. 2012;91(4):413-9.

4. Kaneko K, Kawai S. [Glucocorticoid and Bone. Significance of serum soluble RANKL measurement in patients under glucocorticoid therapy]. Clin Calcium. 2014;24(9):1361-70.

5. Cullinane DM. The role of osteocytes in bone regulation: mineral homeostasis versus mechanoreception. J Musculoskelet Neuronal Interact. 2002;2(3):242-4.

6. Rubin CT, Lanyon LE. Regulation of bone formation by applied dynamic loads. J Bone Joint Surg Am. 1984;66(3):397-402.
7. Andrade I, Jr., Taddei SRA, Garlet GP, Garlet TP, Teixeira AL, Silva TA, et al. CCR5 Down-regulates Osteoclast Function in Orthodontic Tooth Movement. J Dent Res. 2009;88(11):1037-41.

8. Teixeira CC, Khoo E, Tran J, Chartres I, Liu Y, Thant LM, et al. Cytokine expression and accelerated tooth movement. J Dent Res. 2010;89(10):1135-41.

9. Alikhani M, Raptis M, Zoldan B, Sangsuwon C, Lee YB, Alyami B, et al. Effect of micro-osteoperforations on the rate of tooth movement. Am J Orthod Dentofacial Orthop. 2013;144(5):639-48.

10. de Albuquerque Taddei SR, Queiroz-Junior CM, Moura AP, Andrade I, Jr., Garlet GP, Proudfoot AE, et al. The effect of CCL3 and CCR1 in bone remodeling induced by mechanical loading during orthodontic tooth movement in mice. Bone. 2013;52(1):259-67.

11. Bassett CA. Biologic significance of piezoelectricity. Calcif Tissue Res. 1968;1(4):252-72

12. Hert J, Liskova M, Landrgot B. Influence of the long-term, continuous bending on the bone. An experimental study on the tibia of the rabbit. Folia Morphol (Praha). 1969;17(4):389-99.

13. Garman R, Rubin C, Judex S. Small oscillatory accelerations, independent of matrix deformations, increase osteoblast activity and enhance bone morphology. PLoS One. 2007;2(7):e653.

14. Alikhani M, Nervina J, Teixeira C. Biphasic Theory of Tooth Movement: Cytokine Expression and Rate of Tooth Movement In: Shroff B, editor. Biology of Orthodontic Tooth Movement : Current Concepts and Applications in Orthodontic Practice. Switzerland Springer International Publishing; 2016. p. 45-65.

15. Charles JF, Aliprantis AO. Osteoclasts: more than 'bone eaters'. Trends Mol Med. 2014;20(8):449-59.

16. Zahrowski JJ, Turley PK. Force magnitude effects upon osteoprogenitor cells during premaxillary expansion in rats. Angle Orthod. 1992;62(3):197-202.

17. Cox PG, Rayfield EJ, Fagan MJ, Herrel A, Pataky TC, Jeffery N. Functional evolution of the feeding system in rodents. PLoS One. 2012;7(4):e36299.

18. Erben RG. Embedding of bone samples in methylmethacrylate: an improved method suitable for bone histomorphometry, histochemistry, and immunohistochemistry. J Histochem Cytochem. 1997;45(2):307-13.

19. Dahlberg G. Statistical Methods for Medical and Biological Students. $\mathrm{Br}$ Med J. 1940;2(4158):358--9.

20. Houston WJ. The analysis of errors in orthodontic measurements. Am J Orthod. 1983;83(5):382-90.

21. Oliveira SM, Ringshia RA, Legeros RZ, Clark E, Yost MJ, Terracio L, et al. An improved collagen scaffold for skeletal regeneration. J Biomed Mater Res A. 2010;94(2):371-9.

22. Alikhani M AB, Lee I. S. , Almoammar S, Vongthongleur T, Alikhani M, Alansari S, Sangsuawon C, Chou M, Khoo E, Boskey A, Teixeia C. Biological saturation point during orthodontic tooth movement. Orthodontic Craniofacial research. 2015;18(1):8-17.

23. Alikhani M, Alansari S, Sangsuwon C, Alikhani M, Chou MY, Alyami B, et al. Micro-osteoperforations: Minimally invasive accelerated tooth movement. Semin Orthod. 2015;21(3):162-9.

24. Henriksen K, Neutzsky-Wulff AV, Bonewald LF, Karsdal MA. Local communication on and within bone controls bone remodeling. Bone. 2009;44(6):1026-33.

25. Matsuo K, Irie N. Osteoclast-osteoblast communication. Arch Biochem Biophys. 2008;473(2):201-9.

26. Sims NA, Vrahnas C. Regulation of cortical and trabecular bone mass by communication between osteoblasts, osteocytes and osteoclasts. Arch Biochem Biophys. 2014;561:22-8. 
27. Haas AJ. Palatal expansion: just the beginning of dentofacial orthopedics. Am J Orthod. 1970;57(3):219-55.

28. Bishara SE, Staley RN. Maxillary expansion: clinical implications. Am J Orthod Dentofacial Orthop. 1987;91(1):3-14.

29. Boryor A, Hohmann A, Wunderlich A, Geiger M, Kilic F, Kim KB, et al. Use of a modified expander during rapid maxillary expansion in adults: an in vitro and finite element study. Int J Oral Maxillofac Implants. 2013;28(1):e11-6.

30. Cleall JF, Bayne DI, Posen JM, Subtelny JD. EXPANSION OF THE MIDPALATAL SUTURE IN THE MONKEY. Angle Orthod. 1965;35:23-35.

31. Sander C, Huffmeier S, Sander FM, Sander FG. Initial results regarding force exertion during rapid maxillary expansion in children. J Orofac Orthop. 2006;67(1):19-26.

32. Timms DJ. A study of basal movement with rapid maxillary expansion. Am J Orthod. 1980;77(5):500-7.

33. Yepes E, Quintero P, Rueda ZV, Pedroza A. Optimal force for maxillary protraction facemask therapy in the early treatment of class III malocclusion. Eur J Orthod. 2014;36(5):586-94.

34. Iseri H, Tekkaya AE, Oztan O, Bilgic S. Biomechanical effects of rapid maxillary expansion on the craniofacial skeleton, studied by the finite element method. Eur J Orthod. 1998;20(4):347-56.

35. Jafari A, Shetty KS, Kumar M. Study of stress distribution and displacement of various craniofacial structures following application of transverse orthopedic forces--a three-dimensional FEM study. Angle Orthod. 2003;73(1):12-20.

36. Gautam P, Valiathan A, Adhikari R. Stress and displacement patterns in the craniofacial skeleton with rapid maxillary expansion: a finite element method study. Am J Orthod Dentofacial Orthop. 2007;132(1):5.e1-11.

37. Tindlund RS, Rygh P. Maxillary protraction: different effects on facial morphology in unilateral and bilateral cleft lip and palate patients. Cleft Palate Craniofac J. 1993;30(2):208-21.

38. Velazquez P, Benito E, Bravo LA. Rapid maxillary expansion. A study of the long-term effects. Am J Orthod Dentofacial Orthop. 1996;109(4):361-7.

39. Walter CD. Secondary nasal revisions after rhinoplasties. Transactions Section on Otolaryngology American Academy of Ophthalmology and Otolaryngology. 1975;80(6):519-26.

40. Boyer DM, Seiffert ER, Gladman JT, Bloch JI. Evolution and allometry of calcaneal elongation in living and extinct primates. PLoS One. 2013;8(7):e67792.

41. Vardimon AD, Graber TM, Voss LR, Verrusio E. Magnetic versus mechanical expansion with different force thresholds and points of force application. Am J Orthod Dentofacial Orthop. 1987;92(6):455-66.

42. Elder JR, Tuenge RH. Cephalometric and histologic changes produced by extraoral high-pull traction to the maxilla in Macaca mulatta. Am J Orthod.66(6):599-617.

43. Holberg C, Rudzki-Janson I. Stresses at the cranial base induced by rapid maxillary expansion. Angle Orthod. 2006;76(4):543-50.

44. Bell WH, Epker BN. Surgical-orthodontic expansion of the maxilla. Am J Orthod. 1976;70(5):517-28.

45. Capelozza Filho L, De Almeida AM, Ursi WJ. Rapid maxillary expansion in cleft lip and palate patients. J Clin Orthod. 1994;28(1):34-9.

46. Nicholson PT, Plint DA. A long-term study of rapid maxillary expansion and bone grafting in cleft lip and palate patients. Eur J Orthod. 1989;11(2):186-92.

47. Pan X, Qian Y, Yu J, Wang D, Tang Y, Shen G. Biomechanical effects of rapid palatal expansion on the craniofacial skeleton with cleft palate: a threedimensional finite element analysis. Cleft Palate Craniofac J. 2007;44(2):149-54.
48. Liu W, Zhang X. Receptor activator of nuclear factor-kappaB ligand (RANKL)/RANK/osteoprotegerin system in bone and other tissues (review). Mol Med Rep. 2015;11(5):3212-8.

49. Humphrey MB, Nakamura MC. A Comprehensive Review of Immunoreceptor Regulation of Osteoclasts. Clin Rev Allergy Immunol. 2016;51(1):48-58.

50. Yasuda H. RANKL, a necessary chance for clinical application to osteoporosis and cancer-related bone diseases. World journal of orthopedics. 2013;4(4):207-17.

51. Fox SW, Fuller K, Chambers TJ. Activation of osteoclasts by interleukin-1: divergent responsiveness in osteoclasts formed in vivo and in vitro. J Cell Physiol. 2000;184(3):334-40.

52. Dai SM, Nishioka K, Yudoh K. Interleukin (IL) 18 stimulates osteoclast formation through synovial T cells in rheumatoid arthritis: comparison with IL1 beta and tumour necrosis factor alpha. Ann Rheum Dis. 2004;63(11):1379-86.

53. Polzer K, Joosten L, Gasser J, Distler JH, Ruiz G, Baum W, et al. Interleukin-1 is essential for systemic inflammatory bone loss. Ann Rheum Dis. 2010;69(1):284-90.

54. Hienz SA, Paliwal S, Ivanovski S. Mechanisms of Bone Resorption in Periodontitis. Journal of immunology research. 2015;2015:615486.

55. Baka ZM, Akin M, Ucar FI, Ileri Z. Cone-beam computed tomography evaluation of dentoskeletal changes after asymmetric rapid maxillary expansion. Am J Orthod Dentofacial Orthop. 2015;147(1):61-71.

56. Garrett BJ, Caruso JM, Rungcharassaeng K, Farrage JR, Kim JS, Taylor GD. Skeletal effects to the maxilla after rapid maxillary expansion assessed with cone-beam computed tomography. Am J Orthod Dentofacial Orthop. 2008;134(1):8-9.

57. Burdan F, Rozylo-Kalinowska I, Szumilo J, Dudka J, Klepacz R. Cyclooxygenase inhibitors affect bone mineralization in rat fetuses. Cells Tissues Organs. 2008;187(3):221-32.

58. Abukawa H, Phelps M, Jackson P, Smith RM, Vacanti JP, Kaban LB, et al. Effect of ibuprofen on osteoblast differentiation of porcine bone marrow-derived progenitor cells. J Oral Maxillofac Surg. 2009;67(11):2412-7.

59. Rodriguez M, Lopez I, Munoz J, Aguilera-Tejero E, Almaden Y. FGF23 and mineral metabolism, implications in CKD-MBD. Nefrologia : publicacion oficial de la Sociedad Espanola Nefrologia. 2012;32(3):275-8.

60. Lanigan DT, Mintz SM. Complications of surgically assisted rapid palatal expansion: review of the literature and report of a case. J Oral Maxillofac Surg. 2002;60(1):104-10. 